\title{
Indolent fibrin-associated EBV-positive large B cell lymphoproliferative disorder in a lower extremity aneurysmal hematoma: a case report
}

\author{
Chelsea Hayes • Serhan Alkan • Sumire Kitahara
}

Received: 13 June 2014 / Accepted: 24 July 2014 /Published online: 3 August 2014

(C) Springer-Verlag Berlin Heidelberg 2014

\begin{abstract}
Epstein-Barr virus (EBV) is known to play a key role in the development of several lymphoproliferative disorders (LPD), ranging from indolent lesions to highly aggressive lymphomas. Here, we describe an incidentally detected unique case of a localized EBV-positive large B cell LPD within a popliteal artery aneurysmal hematoma of a 91 -yearold male. Histopathologic examination of an expanding right thigh mass demonstrated organizing hematoma with microscopic clusters of atypical large lymphoid cells with a nongerminal center B cell immunophenotype, a high proliferation rate by Ki-67 staining, and strong positivity for EBV-encoded RNA (EBER) by in situ hybridization. An 18-month followup revealed no evidence of disease progression. The presence of cytologically malignant EBV-positive large B cells supports a LPD, with a differential diagnosis that includes a spectrum of EBV-associated lesions, including diffuse large B cell lymphoma (DLBCL) of the elderly, DLBCL associated with chronic inflammation, as well as a variety of recently described indolent EBV-associated large B cell proliferations occurring in cardiac fibrin thrombi, atrial myxomas, and other unusual sites. This case is the first report of an indolent fibrinassociated EBV-positive LPD occurring in an aneurysmal hematoma of the extremity. This lesion may represent the indolent end of the spectrum of EBV-associated large B cell proliferations, and recognition of such lesions may prevent unnecessary aggressive treatment.
\end{abstract}

Keywords Aneurysmal hematoma $\cdot$ Diffuse large B cell lymphoma $\cdot$ Epstein-Barr virus · Fibrin $\cdot$ Lymphoproliferative disorder

C. Hayes $(\bowtie) \cdot$ S. Alkan $\cdot$ S. Kitahara

Department of Pathology and Laboratory Medicine, Cedars-Sinai Medical Center, 8700 Beverly Blvd., Los Angeles, CA 90048, USA e-mail: chelsea.hayes@cshs.org

\author{
Abbreviations \\ AR- $\quad$ Age-related EBV-associated lymphoprolifera- \\ EBVLPD tive disorder \\ DLBCL Diffuse large B cell lymphoma \\ EBV Epstein-Barr virus \\ LPD Lymphoproliferative disorder
}

\section{Introduction}

Epstein-Barr virus (EBV) is known to play a key role in the development of several lymphoproliferative disorders (LPD), ranging from indolent lesions to highly aggressive lymphomas [1]. Interestingly, several cases of EBVpositive large $\mathrm{B}$ cell proliferations arising in cardiac fibrin thrombi, atrial myxomas, pseudocysts, and associated with artificial materials have recently been described. These cases were reported to be of nongerminal center B cell lineage and contained highgrade cytology, high proliferation rate, and EBV positivity; however, all showed a favorable clinical course [2-7].

In this report, we describe a case of a fibrin-associated EBV-positive large B cell LPD localized within a popliteal artery aneurysmal hematoma. Due to its indolent clinical course, unusual site, and microscopic extent of involvement, this lesion does not fit the definition or clinical features of the currently available EBVassociated LPD categories, such as diffuse large B cell lymphoma (DLBCL) of the elderly or DLBCL associated with chronic inflammation. Alternative differential diagnoses, including the recently reported indolent EBVassociated large B cell proliferations occurring in cardiac fibrin thrombi, atrial myxomas, and other unusual sites, are discussed. 


\section{Clinical history}

A 91-year-old Caucasian male presented with an expanding right thigh mass. Approximately 6 years prior, the patient underwent a right below the knee amputation secondary to a ruptured popliteal artery aneurysm occurring at the site of a prior femoral-popliteal artery bypass graft. The patient's past medical history includes a remote diagnosis of Kawasaki's disease with multiple systemic aneurysms, including coronary artery aneurysms complicated by myocardial infarction necessitating coronary artery bypass surgery approximately 30 years ago. There were no reported fevers, night sweats or weight loss, and no prior history of lymphoma.

The patient underwent surgical exploration of the right thigh with resultant evacuation and plication of the right popliteal artery aneurysm and adjacent hematoma. The surgery concluded without complication.

\section{Materials and methods}

The surgical specimen was fixed in $10 \%$ neutral buffered formalin and processed routinely. Paraffin-embedded blocks were sectioned ( $4 \mu \mathrm{m}$ thick) and stained with hematoxylin and eosin. Immunohistochemical analysis was carried out with a BenchMark ULTRA system (Ventana, Tucson, AZ), according to the manufacturer's instructions, using the following primary antibodies: CD45 (2B11/PD7126, Ventana, Tucson, AZ), CD20 (L26, Dako, Carpinteria, CA), CD3 (LN10, Leica, Buffalo Grove, IL), Ki-67 (30-9, Ventana), CD10 (56C6, Leica), BCL6 (GI191E/A8, Cell Marque, Rocklin, CA), MUM1 (MRQ-43, Ventana), BCL2 (124, Ventana), CD30 (BER-H2, Dako), CD5 (4C7, Cell Marque), p53 (DO7, Ventana), BCL1 (SP4, Cell Marque), CD43 (L60, Ventana), AE1/AE3 (Leica), S-100 (Polyclonal, Leica), and vimentin (SRL33, Leica). Additionally, EBER in situ hybridization (EBER1 DNP Probe, Ventana) was performed.

\section{Results}

On gross examination, the specimen consisted of a $15-\mathrm{cm}$ aggregate of multiple fragments of red-brown friable blood clot. Histologic sections showed predominantly organizing hematoma with fibrin and acellular material. However, in rare microscopic foci, small sheets of cytologically malignantappearing large lymphoid cells with ovoid to irregular nuclei, vesicular chromatin, single to multiple nucleoli, as well as moderate amounts of cytoplasm (Fig. 1a) were present, which made up less than $5 \%$ of submitted material. Foci of necrosis and apoptotic cells were noted adjacent to the atypical lymphoid cells.
Immunohistochemically, the atypical lymphoid cells were positive for CD45, CD20 (Fig. 1b), MUM1, CD30, and CD43 and were partially positive for BCL- 2 and p 53 . Ki-67 revealed a proliferation rate of approximately $90 \%$ (Fig. 1c). Of particular interest, the atypical lymphoid cells were also strongly positive for EBV-encoded RNA (EBER) by in situ hybridization (Fig. 1d), as well as MYC by immunohistochemistry. Staining for EBV-LMP, HHV8, CD3, CD5, CD10, BCL-1, and BCL-6 was negative. The pathologic differential diagnosis included a spectrum of EBV-associated LPDs but was particularly concerning for an aggressive lymphoma due to the presence of cytologically malignantappearing $\mathrm{EBV}+\mathrm{B}$ cells.

Following 18 months of clinical follow-up in which no additional treatment was given, the patient has shown no evidence of disease progression or lymphadenopathy by physician examination. The surgical site demonstrated complete wound healing with no signs or symptoms of infection (Fig. 2).

\section{Discussion}

Knowledge regarding the clinicopathologic spectrum of lymphoid lesions demonstrating EBV infection has been greatly expanded in the recent years. EBV, an ubiquitous $\gamma$-herpes virus, plays a key role in the development of several B cell LPDs and lymphomas [8-10]. The clinical behavior of the EBV-associated lesions varies from indolent and self-limited, such as EBV lymphadenitis, to highly aggressive lymphomas, such as Burkitt's lymphoma. A spectrum of EBV-driven LPDs occur in patients who are immunosuppressed, either due to primary immune deficiency, HIV infection, chronic inflammatory states, posttransplant immunosuppression, or iatrogenic immunosuppression, as well as a more recently described immunosenescence related to aging, which encompasses the age-related EBV-associated LPDs [9].

After review of the available literature, this case is thought to represent the first report of an EBV-associated large B cell LPD occurring in an aneurysmal hematoma of the extremity. Based on the patient's age, immunocompetent status, and medical history, the diagnostic possibilities for this lesion include age-related EBV-associated LPD (AR-EBVLPD), such as DLBCL of the elderly, as well as DLBCL associated with chronic inflammation, or an indolent fibrin-associated EBV-positive LPD similar to the cases recently described in cardiac fibrin thrombi and atrial myxomas [2-7, 11-13].

AR-EBVLPDs as a group represent a wide clinicopathologic spectrum, including reactive hyperplasia, polymorphic nodal and extranodal LPD, and DLBCL of the elderly, with a corresponding increase in aggressive clinical behavior $[9,10]$. On one end of the spectrum, indolent lesions exist, such as EBV-associated mucocutaneous ulcers, which represent a 
Fig. 1 Histopathologic and immunohistochemical findings. a Small sheets of atypical, medium to large lymphoid cells characterized by round to irregular nuclei, vesicular chromatin, single to multiple nucleoli, as well as moderate amounts of cytoplasm are seen among a predominating background of organizing hematoma. b The lymphoid cells show strong reactivity for CD20. c The proliferation rate by $\mathrm{Ki}-67$ staining is approximately $90 \%$. d The lymphoid cells were also strongly positive for Epstein-Barr virus (EBV) by EBER in situ hybridization

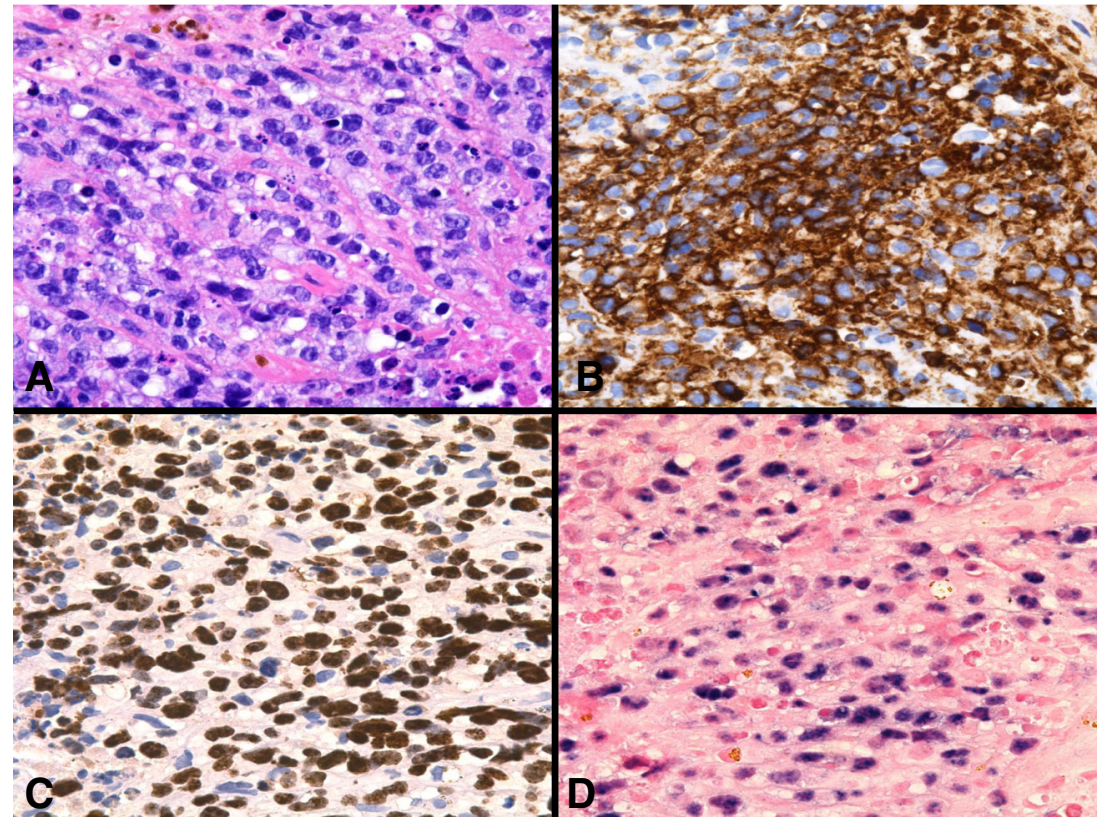

well-circumscribed, isolated ulcer of the skin or oropharyngeal mucosa, show a polymorphous infiltrate, and demonstrate a high rate of spontaneous remission with no reported disease-associated deaths [9]. Although the current lesion is also indolent in nature, the monomorphic morphology and non-mucocutaneous location argue against this diagnosis. At the opposite end of the spectrum, EBV-positive DLBCL of the elderly exists, which usually occurs in individuals 50 years of

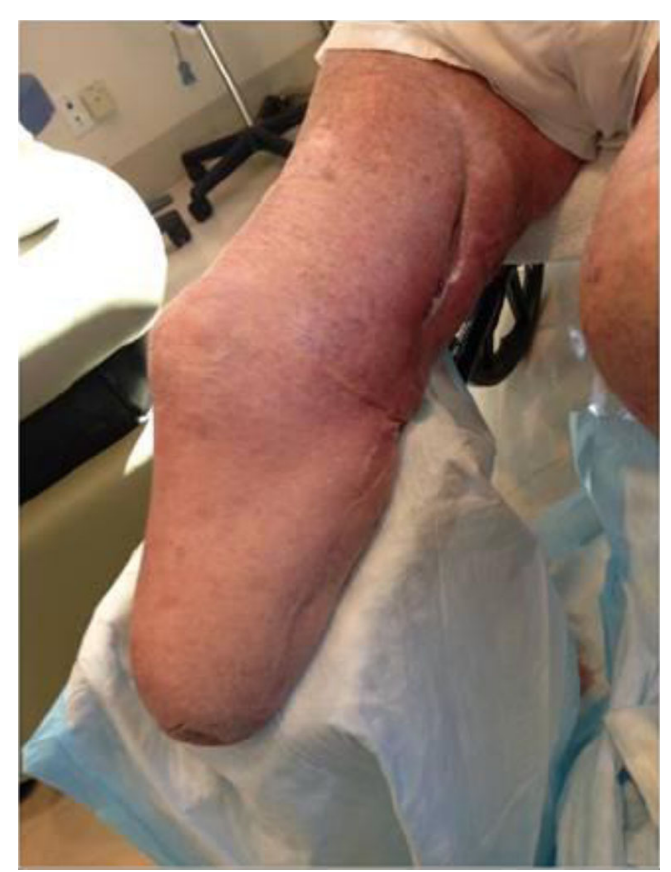

Fig. 2 Right thigh surgical site demonstrating complete wound healing and no signs or symptoms of infection age or greater and has been shown to represent an aggressive $\mathrm{B}$ cell neoplasm with poor overall survival $[8,9,14]$. While the current lesion contains histologic features of a high-grade process, the apparent indolent clinical course argues against a diagnosis of DLBCL of the elderly.

DLBCL associated with chronic inflammation is also an aggressive lymphoma, most commonly involving body cavities or enclosed sites, with pyothorax-associated lymphoma being the prototypical example. Most cases of pyothoraxassociated lymphoma have been reported in Japanese patients who have undergone artificial pneumothorax as a form of surgical therapy for tuberculosis $[8,15]$. This entity has also been described in association with other chronic inflammatory conditions, such as osteomyelitis [16-18], metallic prosthetic implants [19-23], as well as other foreign materials, such as polyethylene terephthalate (PET) surgical mesh [7, 24]. The current patient does have a history of prior femoral-popliteal artery bypass grafting using polytetrafluoroethylene (PTFE) surgical mesh at the site of interest; however, this type of mesh is different than the PET mesh previously reported to be associated with DLBCL, and the relationship between the current lesion and the mesh material is unclear. Additionally, although the patient has a remote history of Kawasaki disease, which is described as an acute inflammatory vasculitis [25], the pathologic specimen demonstrated no evidence of suppurative inflammation and very little chronic inflammation. It is important to note that assessment for inflammation within the surrounding tissue was not possible because the aneurysm wall was not resected. Given that DLBCL associated with chronic inflammation arises in the context of long-standing inflammation, the lack of significant amounts of inflammation in the current specimen and its indolent clinical course argue 
against this diagnosis. Additionally, there are very few case reports describing the development of lymphoproliferative disorders following a diagnosis of Kawasaki disease. Most of these cases were acute lymphocytic leukemia, and all cases developed only weeks following the initial diagnosis of Kawasaki disease [26].

Prior studies have postulated that the pathogenesis of DLBCL associated with chronic inflammation is due to the local immunosuppression caused by cytokines at the site of inflammation. In addition to local immunosuppression, it is thought that occurrence in a closed environment allows EBVinfected $B$ cells to escape immune surveillance and proliferate [7].

Finally, the literature contains several recent reports describing indolent, EBV-positive large B cell proliferations occurring in a variety of anatomic sites, including atrial myxomas, cardiac fibrin thrombi, adrenal gland cysts, paratesticular pseudocysts, splenic false cysts, renal cysts, and hydroceles [7, 11, 27-29]. Similar to the current case, five cases of $\mathrm{EBV}+\mathrm{B}$ cell proliferations arising in atrial myxomas were of B cell lineage and EBV-positive, contained high-grade cytology with a high proliferation rate, and were associated with a favorable course $[2-5,7]$. Additionally, six of the eight cases of DLBCL detected within cardiac fibrin thrombi without myocardial involvement $[6,11-13]$ were found to be EBV-positive, and all cases occurred in immunocompetent individuals without evidence of systemic disease and with favorable outcomes. Several possible mechanisms regarding the development of DLBCL associated with cardiac fibrin thrombi have been postulated, including a primary neoplastic process occurring in and confined to a previously formed thrombus, a remote lymphoma seeding a previously formed thrombus, as well as initial involvement of the cardiac wall followed by spontaneous regression [11]. Aside from the location, the current case is strikingly similar to these fibrinassociated cardiac lymphomas and bears resemblance to the reports of cystic lesions in which only microscopic foci of EBV-positive DLBCL were identified [7, 27, 29].

In summary, we do not favor classification of this entity as an aggressive subtype of DLBCL, such as EBV-positive DLBCL of the elderly or DLBCL associated with chronic inflammation. Although the cytology and immunophenotype resemble those of an aggressive lymphoma, the clinical course has been indolent with complete wound healing, lack of lymphadenopathy, and no evidence of disease progression after 18 months. We believe that additional categories of EBV-positive LPDs may exist, particularly a monomorphic large cell proliferation with a favorable clinical course, such as the current case and those described in cardiac fibrin thrombi and atrial myxomas. This lesion may represent the indolent end of the spectrum of EBV-associated large B cell proliferations, and recognition of such lesions may prevent unnecessary aggressive treatment.

Conflict of interest The authors have no conflict of interest to declare.

\section{References}

1. Grywalska E, Markowicz J, Grabarczyk P, Pasiarski M, Rolinski J (2013) Epstein-Barr virus-associated lymphoproliferative disorders. Postepy Hig Med Dosw (Online) 67:481-490

2. Svec A, Rangaiah M, Giles M, Jaksa R, McAulay KA (2012) EBV+ diffuse large B-cell lymphoma arising within atrial myxoma. An example of a distinct primary cardiac EBV+DLBCL of immunocompetent patients; Pathol res pract. Germany, 2012 Elsevier GmbH, 2012, vol 208, pp 172-176.

3. Bagwan IN, Desai S, Wotherspoon A, Sheppard MN (2009) Unusual presentation of primary cardiac lymphoma. Interact Cardiovasc Thorac Surg 9:127-129

4. Dimitrova KR, Hoffman DM, Geller CM, Thiagarjah P, Master J, Berger M, Tranbaugh RF (2010) Malignant B-cell lymphoma arising in a large, left atrial myxoma. Ann Thorac Surg 89:626-629

5. White RW, Hirst NA, Edward S, Nair UR (2010) Plasmacytoid lymphoma within a left atrial myxoma: a rare coincidental dual pathology. Interact Cardiovasc Thorac Surg 10:140-141

6. Gruver AM, Huba MA, Dogan A, Hsi ED (2012) Fibrin-associated large B-cell lymphoma: part of the spectrum of cardiac lymphomas. Am J Surg Pathol 36:1527-1537

7. Loong F, Chan AC, Ho BC, Chau YP, Lee HY, Cheuk W, Yuen WK, Ng WS, Cheung HL, Chan JK (2010) Diffuse large B-cell lymphoma associated with chronic inflammation as an incidental finding and new clinical scenarios. Mod Pathol 23:493-501

8. Dunleavy K, Roschewski M, Wilson WH (2012) Lymphomatoid granulomatosis and other Epstein-Barr virus associated lymphoproliferative processes. Curr Hematol Malig Rep 7:208-215

9. Dojcinov SD, Venkataraman G, Pittaluga S, Wlodarska I, Schrager JA, Raffeld M, Hills RK, Jaffe ES (2011) Age-related ebv-associated lymphoproliferative disorders in the western population: a spectrum of reactive lymphoid hyperplasia and lymphoma. Blood 117:47264735

10. Oyama T, Ichimura K, Suzuki R, Suzumiya J, Ohshima K, Yatabe Y, Yokoi T, Kojima M, Kamiya Y, Taji H, Kagami Y, Ogura M, Saito H, Morishima Y, Nakamura S (2003) Senile EBV+B-cell lymphoproliferative disorders: a clinicopathologic study of 22 patients. Am J Surg Pathol 27:16-26

11. Quigley MM, Schwartzman E, Boswell PD, Christensen RL, Gleason LA, Sharpe RW, D'Amato TA (2003) A unique atrial primary cardiac lymphoma mimicking myxoma presenting with embolic stroke: a case report. Blood 101:4708-4710

12. Miller DV, Firchau DJ, McClure RF, Kurtin PJ, Feldman AL (2010) Epstein-Barr virus-associated diffuse large B-cell lymphoma arising on cardiac prostheses. Am J Surg Pathol 34:377-384

13. Durrleman NM, El-Hamamsy I, Demaria RG, Carrier M, Perrault LP, Albat B (2005) Cardiac lymphoma following mitral valve replacement. Ann Thorac Surg 79:1040-1042

14. Shimoyama Y, Oyama T, Asano N, Oshiro A, Suzuki R, Kagami Y, Morishima Y, Nakamura S (2006) Senile Epstein-Barr virus-associated B-cell lymphoproliferative disorders: a mini review. J Clin Exp Hematop 46:1-4

15. Aozasa K (2006) Pyothorax-associated lymphoma. J Clin Exp Hematop 46:5-10 
16. Copie-Bergman C, Niedobitek G, Mangham DC, Selves J, Baloch K, Diss TC, Knowles DN, Delsol G, Isaacson PG (1997) Epstein-Barr virus in B-cell lymphomas associated with chronic suppurative inflammation. J Pathol 183:287-292

17. Posnett DN, Collins RD, Krantz SB (1979) Osteomyelitis and lymphoma. Lancet 2:1085, England

18. Laurent G, Pris J, Delsol G, Familiades J, Fabre J (1980) Immunoblastic lymphoma and osteomyelitis. Lancet 1:258, England

19. McDonald I (1981) Malignant lymphoma associated with internal fixation of a fractured tibia. Cancer 48:1009-1011

20. Dodion P, Putz P, Amiri-Lamraski MH, Efira A, de Martelaere E, Heimann R (1982) Immunoblastic lymphoma at the site of an infected vitallium bone plate. Histopathology 6:807-813

21. Ito H, Shimizu A (1999) Malignant lymphoma at the site of a total hip replacement. Orthopedics 22:82-84

22. Ganapathi M, Lake DN, Griffiths AP (2001) Periprosthetic highgrade B-cell lymphoma complicating an infected revision total hip arthroplasty. J Arthroplasty 16:229-232

23. Syed AA, Agarwal M, Fenelon G, Toner M (2002) Osseous malignant non-Hodgkin's B-cell lymphoma associated with total hip replacement. Leuk Lymphoma 43:2213-2216
24. Fujimoto M, Haga H, Okamoto M, Obara E, Ishihara M, Mizuta N, Nishimura K, Manabe T (2008) EBV-associated diffuse large B-cell lymphoma arising in the chest wall with surgical mesh implant. Pathol Int 58:668-671

25. Bajolle F (2012) Laux D: [Kawasaki disease: what you need to know]. Arch Pediatr 19:1264-1268

26. Suzuki H, Takeuchi T, Minami T, Shima Y, Iwahashi S, Shibuta S, Uemura S, Kounami S, Aoyagi N, Yoshikawa N (2005) Neoplasms in three patients following Kawasaki disease. Pediatr Int 47:217-219

27. Valli R, Piana S, Capodanno I, Cavazza A (2011) Diffuse large B-cell lymphoma associated with chronic inflammation arising in a renal pseudocyst. Int J Surg Pathol 19:117-119

28. Cheuk W, Chan AC, Chan JK, Lau GT, Chan VN, Yiu HH (2005) Metallic implant-associated lymphoma: a distinct subgroup of large B-cell lymphoma related to pyothorax-associated lymphoma? Am J Surg Pathol 29:832-836

29. Boroumand N, Ly TL, Sonstein J, Medeiros LJ (2012) Microscopic diffuse large B-cell lymphoma (DLBCL) occurring in pseudocysts: do these tumors belong to the category of DLBCL associated with chronic inflammation? Am J Surg Pathol 36:1074-1080 